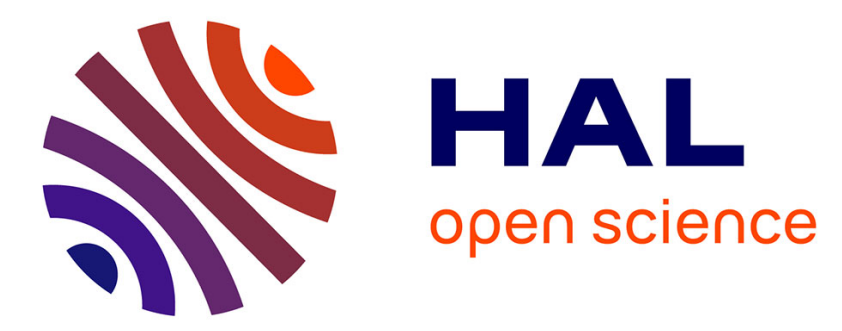

\title{
Role of the charge transfer state in the electronic absorption of protonated hydrocarbon molecules
}

Ivan Alata, Claude Dedonder, Michel Broquier, Ernesto Marceca, Christophe Jouvet

\section{To cite this version:}

Ivan Alata, Claude Dedonder, Michel Broquier, Ernesto Marceca, Christophe Jouvet. Role of the charge transfer state in the electronic absorption of protonated hydrocarbon molecules. 2010. inria00505242

\section{HAL Id: inria-00505242 \\ https://hal.inria.fr/inria-00505242}

Preprint submitted on 23 Jul 2010

HAL is a multi-disciplinary open access archive for the deposit and dissemination of scientific research documents, whether they are published or not. The documents may come from teaching and research institutions in France or abroad, or from public or private research centers.
L'archive ouverte pluridisciplinaire HAL, est destinée au dépôt et à la diffusion de documents scientifiques de niveau recherche, publiés ou non, émanant des établissements d'enseignement et de recherche français ou étrangers, des laboratoires publics ou privés. 
Role of the charge transfer state in the electronic absorption of protonated hydrocarbon molecules

\author{
I. Alata ${ }^{1,3}$, C. Dedonder ${ }^{1,2}$, M. Broquier ${ }^{1,2}$, E. Marceca ${ }^{4}$, C. Jouvet ${ }^{1,2}$ * \\ 1) Centre Laser de l'Université Paris Sud (LUMAT FR 2764) Bât. 106, Univ. Paris-Sud \\ 91405 Orsay Cedex, France
}

2) Institut des Sciences Moléculaires d'Orsay Bât. 210, Univ. Paris-Sud 91405 Orsay Cedex,

France

3) Atomic Energy Commission of Syria, Damascus, P.O. Box 6091 Syria. http://www.aec.org.sy/

4) INQUIMAE-UBA, Ciudad. Universitaria, 3er piso, Pab. II, (1428) Buenos Aires, Argentina

\begin{abstract}
The vibrationally resolved electronic spectra of isolated protonated polycyclic aromatic hydrocarbons - naphthalene, anthracene, tetracene - have been recorded via neutral photofragment spectroscopy. The $\mathrm{S}_{1} \leftarrow \mathrm{S}_{0}$ transitions are all in the visible region and do not show a monotonic red shift as a function of the molecular size as observed for the neutral analogues. Comparison with ab initio calculations indicates that this behaviour is due to the nature of the excited state, which has a pronounced charge transfer character for protonated linear PAH with an even number of aromatic rings.
\end{abstract}

\footnotetext{
*Corresponding author: christophe.jouvet@u-psud.fr
} 


\section{Introduction}

Very little is known about the structure, dynamics, and electronic properties of protonated molecules, of even simple isolated polyaromatic protonated $\mathrm{AH}^{+}$ions. Recent advances in the development of efficient ion sources, sensitive spectroscopic detection and ion trapping techniques have allowed substantial progress in the characterization of the ground state geometric structure of isolated and microsolvated $\mathrm{AH}^{+}$ions in the gas phase $\mathrm{e}^{1-5}$.

We have recently started to investigate the electronic structure and excited states properties of very simple protonated aromatic molecules in order to get some information on these species which are present the various environments. The knowledge of their spectroscopy is a necessary step in order to detect these species in remote environments such as molecular interstellar clouds, planetary atmosphere, plasmas or jet engine exhaust.

We have shown that the excited state properties of these excited protonated molecules is not simple to predict. From ab initio calculations it appears that the excited states of protonated benzene are unstable and probably undergo a very fast internal conversion. ${ }^{6}$ This is due to a very strong change in geometry in the excited state, the system looses its planar symmetry upon electronic excitation and conical intersections between the first excited states and the ground state arise along out of plane bending coordinates. This seems substantiated by the experimental results since we have been able to record well structured excitation spectra for protonated molecules like benzaldehyde ${ }^{7}$ and naphthalene but not for benzene.

Compared to their neutral analogues, the protonated molecules in their excited states seem to be shorter lived, as it is the case for protonated aromatic amino acids. ${ }^{8-10}$ This is particularity true in the tryptophan case where the excited state lifetime is in the femtosecond domain for the protonated species and in the nanosecond domain for neutral tryptophan. It should be noticed that if protonation has a strong influence on the excited state lifetimes of these aromatic amino acids, the change in the excited state transition energy is very small. ${ }^{10,11}$

Recently we reported the first observation of the excitation spectrum of protonated benzene dimer. ${ }^{12}$ The observed transition is around $450 \mathrm{~nm}$, largely red shifted in comparison with the neutral dimer transition. This transition is also strongly red shifted with respect to the protonated benzene transition. ${ }^{13}$ This strong red-shift is due to the charge transfer character of the first excited state, where an electron of the highest $\pi$ orbital localized on the neutral benzene moiety is transferred to a $\pi^{*}$ orbital localized on the protonated benzene part.

In the present paper we would like to address the following questions on simple protonated aromatic molecules, the linear Polycyclic Aromatic Hydrocarbons (PAH). 
1) In neutral PAHs, the electronic absorption scales with the number of the aromatic cycles. As the size of the molecule increases the electron are more and more delocalized and in the simple "particle in the box" picture, the larger the box, the smaller the energy gap between the quantum states. Is this also valid for the protonated species?

2) The excited state lifetime of protonated PAHs. There is an intrinsic relation between the excited state geometry change and the excited state lifetime. Indeed, large geometry changes lead quite often to conical intersections with the ground state thus to very short lifetimes as for example in DNA bases. ${ }^{14,15}$ This is the case for protonated benzene in which calculations predict a conical intersection between $S_{1}$ and $S_{0}$, and no vibrational structure can be recorded because the excited state lifetime is expected to be very short. In contrast, in protonated naphthalene the ground and excited state geometries are more similar and vibrationally resolved photofragmentation spectra have been obtained. What about larger PAHs?

3) What is the nature of the excited state? In the protonated benzene dimer and in protonated naphthalene, the excited state has a strong charge transfer character. Is it also true for larger PAHs?

We are presenting the first experimental excitation spectra of protonated linear PAHs anthracene and tetracene that will be compared to protonated naphthalene. As we will see, the non-monotonic shift of the first electronic transition with the number of aromatic cycles can be understood considering the character of the excited states (charge transfer state or not) evidenced through ab initio calculations.

\section{Experimental}

The experimental setup has been described previously ${ }^{12,16}$ and is detailed in the supplementary information. Protonated PAH ions are produced by a discharge ignited in a pulsed supersonic expansion in a mixture of $\mathrm{He}$ and $\mathrm{H}_{2}$. Since the large PAH have to be heated at $250^{\circ} \mathrm{C}$ in order to get enough vapour pressure to perform the experiment, a modified pulsed expansion is used following the design of Pr T. Ebata. ${ }^{17}$ The cold ions are extracted and accelerated in a reflectron time-of-flight mass spectrometer.

The mass resolution is good enough to discriminate between the radical cation and the protonated ion and to guarantee that the laser is exciting selectively the protonated species. (see supplementary information). Using a high proportion of $\mathrm{H}_{2}$ in the expansion, it is 
possible to have a concentration of protonated species ten times larger than that of radical cations.

The mass-selected ions are resonantly photofragmented with photons generated by an optical parametric oscillator laser. Resulting fast neutral fragments are detected by a multichannel plate detector, whereas all the ions (parents or fragments) are repelled by an electrostatic field. The fragmentation region (intersection between the ion bunch and the laser) is held at a high voltage or high electric field to evaluate the fragmentation time.

Since our detection relies on the fragmentation of the molecule, the first band in the electronic spectrum may correspond to the fragmentation threshold. If this were the case, one should observe a rather long fragmentation time for the first band and a strong dependence of the fragmentation time on the excess energy. The fragmentation time can be measured when a $1 \mathrm{kV} / \mathrm{cm}$ electric field is applied in the interaction zone. In this case the kinetic energy of the parent ion is changing as it travels inside the interaction zone and the neutral fragment keeps the parent ion velocity. As a result, if the fragmentation time is longer than $10 \mathrm{~ns}$, the ensemble of neutrals emitted during the parent ion lifetime will give rise to a peak broadened towards longer time of flights. With this technique a resolution of $10 \mathrm{~ns}$ can be achieved. ${ }^{18}$

The real difficulty of this experiment is to achieve a good cooling of the protonated ions. First the neutral molecules are produced from a heated oven and then they are heated by the protonation process (probably due to collision with $\mathrm{H}_{3}{ }^{+}$produced by the discharge). Thus many collisions with cold He atoms are necessary to cool down the protonated species. At the same time if there are too many collisions recombination with the electrons present in the plasma generated by the discharge will remove the ions. The production of cold species results then in a delicate balance between too much and not enough collisions. In all the spectra that have been recorded, the vibrational bands are observed superimposed on a continuous background. We assign this background to hot molecules left over from the cooling process and probably the quantity of cold molecules giving the observed structured spectra is just a small portion of the protonated ions produced by the discharge.

When the molecular size increases, the oven temperature has to increase and the initial internal energy of the precursor increases, so that it is more and more difficult to cool down the ions, making the experiment more difficult. 


\section{Calculations.}

$A b$ initio calculations have been performed with the TURBOMOLE ${ }^{\circledR}$ program package, making use of the resolution-of-the-identity (RI) approximation for the evaluation of the electron-repulsion integrals. The equilibrium geometry of the ground electronic state $\left(\mathrm{S}_{0}\right)$ has been determined at the MP2 level. Excitation energies and equilibrium geometry of the lowest excited singlet state $\left(\mathrm{S}_{1}\right)$ have been determined at the RI-CC2 level. Calculations were performed with the def-SVP basis set because of the size of the molecules.

\section{Results and discussion}

\section{a) Experiment}

In figure 1 are presented the photo-fragmentation spectra of protonated naphthalene, anthracene and tetracene. As discussed above, the spectra are composed of sharp vibrational bands issued from cold molecules superimposed on a background issued from hot molecules. Protonated naphthalene and anthracene absorb in the same spectral region whereas protonated tetracene is strongly red shifted. The spectra start at $503.36 \mathrm{~nm}$ for protonated naphthalene, $491.43 \mathrm{~nm}$ for protonated anthracene and $679.90 \mathrm{~nm}$ for protonated tetracene, with an accuracy of $\sim 3 \mathrm{~cm}^{-1}(0.1 \mathrm{~nm})$. 


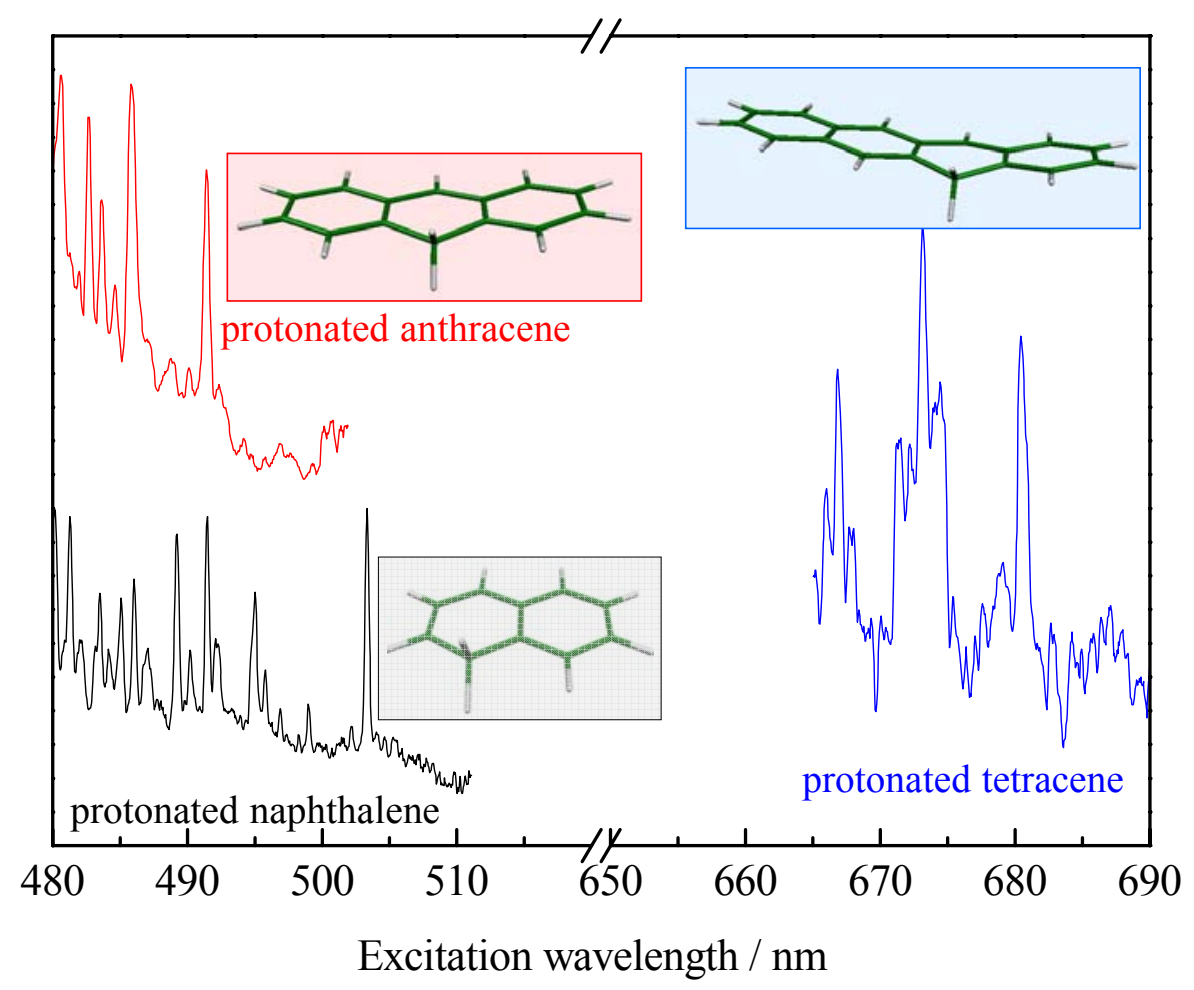

Figure 1. Photofragmentation spectra of protonated linear PAHs. The intensities have been normalized to the first band in each spectrum. The excited state geometries of the molecules are represented in the insets.

As already mentioned, the first band observed in the spectra may correspond to the dissociation threshold. However, when a $1 \mathrm{kV} / \mathrm{cm}$ electric field is applied in the interaction region, there is no broadening of the photo-fragment peak indicating that the neutrals are produced in a few tens of nanosecond for the first band, which seems too short for a fragmentation at threshold.

The dissociation threshold for naphthalene has been calculated at $2.69 \mathrm{eV}^{1}$ and for anthracene, and tetracene, assuming that the main dissociation path is the loss of atomic hydrogen as in IRMPD $^{3}$ the dissociation energy can be evaluated from the thermochemical data $^{19}$ as

$$
\mathrm{E}_{\mathrm{diss}}\left(\mathrm{MH}^{+} \rightarrow \mathrm{M}^{+}+\mathrm{H}\right)=\mathrm{PA}(\mathrm{M})+\mathrm{IP}(\mathrm{M})-\mathrm{IP}(\mathrm{H})
$$

where $\mathrm{PA}(\mathrm{M})$ is the proton affinity of the neutral molecule $\mathrm{M}, \mathrm{IP}(\mathrm{M})$ is its ionization potential, $\mathrm{IP}(\mathrm{H})$ is the ionisation potential of the hydrogen atom. 
This leads to dissociation values of $2.93 \mathrm{eV}$ for anthracene and $2.75 \mathrm{eV}$ for tetracene. In all these cases, the first bands observed in the spectra lie below the dissociation threshold, the spectrum is thus not limited by the fragmentation threshold at low energy and the dissociation is a multiphoton process via the resonant $\mathrm{S}_{1} \leftarrow \mathrm{S}_{0}$ transition.

In a first step we will consider that the first band observed in each spectrum corresponds to the 0-0 transition. Under this hypothesis, strong 0-0 transitions are observed, which implies that the excited state equilibrium geometry is not strongly changed as compared to the ground state geometry in contrast to the benzene case for which the geometry change is predicted to be very important.

In addition the excited state lifetimes are not very short, i.e. longer than $\sim 1 \mathrm{ps}$ in contrast to protonated benzene or tryptophan since the bandwidths are in the order of $10 \mathrm{~cm}^{-1}$, the bandwidths including the rotational envelope.

\section{b) Calculations}

For each system we searched for the most stable isomer in the ground state at the RIMP2 level. The isomers calculated are presented in figure 2. The ground state energies of the different isomers are given in Table 1, the energy being referenced to the most stable isomer. This most stable structure corresponds to the isomer for which the proton is located closer to the middle of the PAH chain as in previous DFT calculations. ${ }^{3}$ Only the most stable isomer of pentacene, with the proton attached to the central ring has been calculated. 


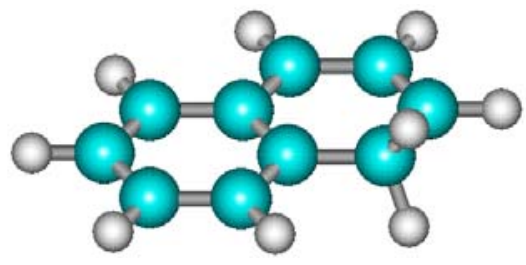

protonated naphthalene Isomer C1

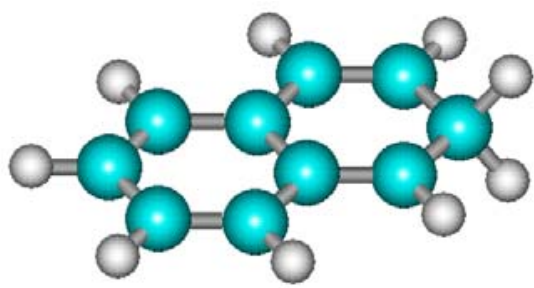

protonated naphthalene Isomer C2

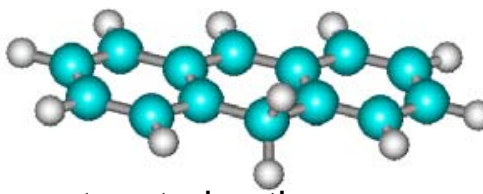

protonated anthracene isomer $\mathrm{C} 1$
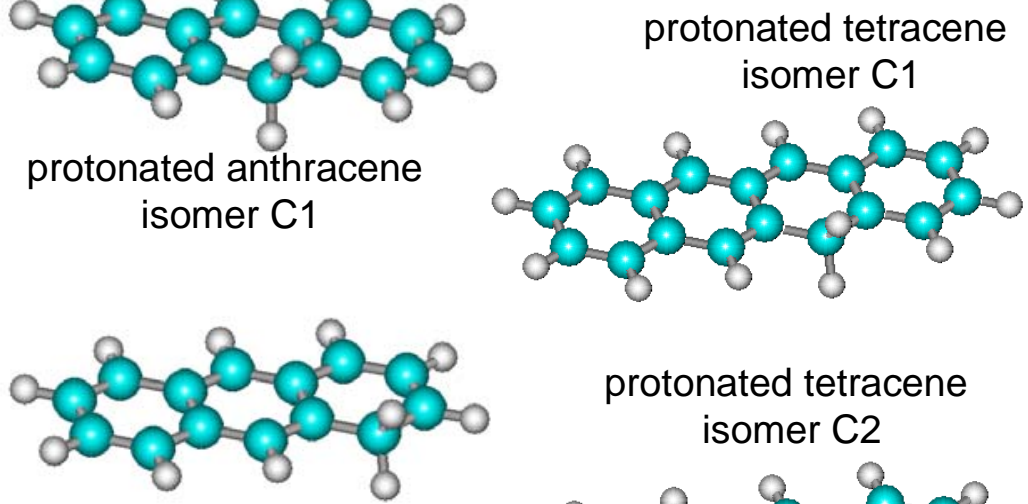

protonated tetracene isomer C2

protonated anthracene isomer $\mathrm{C} 2$
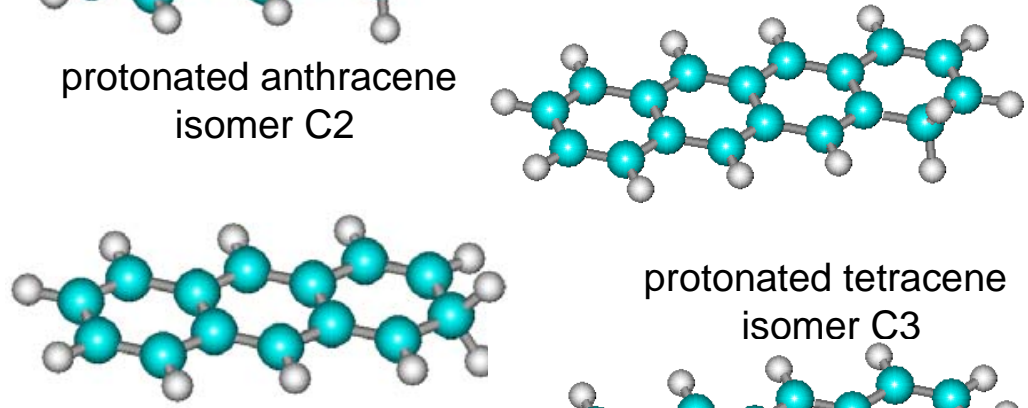

protonated anthracene isomer $\mathrm{C} 3$

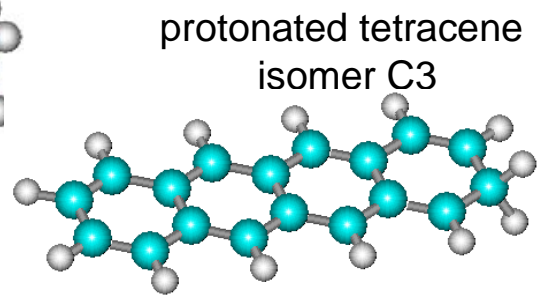

Figure 2: Different isomers calculated for protonated naphthalene, anthracene and tetrasene.

For each isomer the vertical and adiabatic transition energies have been calculated, the latter can be directly compared with the experimental spectrum provided the structural changes between the ground and excited states are not too large so that the 0-0 transition will have a reasonable Franck-Condon factor.

For naphthalene and anthracene, the ground and excited state ZPE have been calculated for the most stable isomer to correct the transition energy.

Table 1. Calculated ground and excited state energies for naphthalene, anthracene and tetracene calculated at the RI-CC2 (def-SVP) level (all the values are given in eV). For pentacene only the most stable Cl isomer with the proton on the central ring has been calculated for comparison with the smaller molecules.

Second column: ground state energy for the different isomers. the energies are referenced to the most stable Cl isomer, which is the one where the proton is closer to the middle of the $P A H$ chain (see figure 2); third and fourth column: vertical excitation energies $\left(S_{1}\right.$ and $S_{2}$ energy at the ground state geometry); Fifth column: calculated adiabatic transition: $S_{1}$ energy 
(after optimization of the $S_{1}$ geometry) minus $S_{0}$ energy (after $S_{0}$ optimization). This value corresponds to the transition energy and can be compared with the experimental band origin given in the first column.

\begin{tabular}{|l|c|c|c|c|}
\hline $\begin{array}{l}\text { NaphthaleneH } \\
\text { Obs=2.47eV }\end{array}$ & $\begin{array}{l}\text { Ground state } \\
\text { energy/C1 }\end{array}$ & $\begin{array}{c}\mathrm{S}_{1} \text { vertical } \\
\text { transition energy }\end{array}$ & $\begin{array}{c}\mathrm{S}_{2} \text { vertical } \\
\text { transition energy }\end{array}$ & $\begin{array}{c}\mathrm{S}_{1} \leftarrow \mathrm{S}_{0} \text { adiabatic } \\
\text { transition energy }\end{array}$ \\
\hline $\mathrm{C} 1$ & 0 & 3.00 & 3.52 & $2.61 / 2.48$ ZPE corrected \\
\hline $\mathrm{C} 2$ & $0.15 / 0.16$ & 2.77 & 4.47 & $2.46 / 2.36$ ZPE corrected \\
\hline
\end{tabular}

\begin{tabular}{|l|c|c|c|c|}
\hline $\begin{array}{l}\text { AnthraceneH } \\
\text { Obs=2.51eV }\end{array}$ & $\begin{array}{l}\text { Ground state } \\
\text { energy/C1 }\end{array}$ & $\begin{array}{c}\mathrm{S}_{1} \text { vertical } \\
\text { transition energy }\end{array}$ & $\begin{array}{c}\mathrm{S}_{2} \text { vertical } \\
\text { transition energy }\end{array}$ & $\begin{array}{c}\mathrm{S}_{1} \leftarrow \mathrm{S}_{0} \text { adiabatic } \\
\text { transition energy }\end{array}$ \\
\hline $\mathrm{C} 1$ & 0 & 2.92 & 3.22 & $2.71 / 2.53$ ZPE corrected \\
\hline C2 & 0.45 & 2.05 & 4.94 & 1.73 \\
\hline C3 & 0.61 & 1.93 & 5.01 & 1.64 \\
\hline
\end{tabular}

\begin{tabular}{|l|c|c|c|c|}
\hline $\begin{array}{l}\text { TetraceneH } \\
\text { Obs=1.82eV }\end{array}$ & $\begin{array}{l}\text { Ground state } \\
\text { energy/C1 }\end{array}$ & $\begin{array}{c}\mathrm{S}_{1} \text { vertical } \\
\text { transition energy }\end{array}$ & $\begin{array}{c}\mathrm{S}_{2} \text { vertical } \\
\text { transition energy }\end{array}$ & $\begin{array}{c}\mathrm{S}_{1} \leftarrow \mathrm{S}_{0} \text { adiabatic } \\
\text { transition energy }\end{array}$ \\
\hline C1 & 0 & 2.152 & 2.97 & 1.90 \\
\hline C2 & 0.64 & 1.48 & 2.84 & 1.19 \\
\hline C3 & 0.79 & 1.39 & 2.88 & 1.13 \\
\hline
\end{tabular}

\begin{tabular}{|c|c|c|c|c|}
\hline PentaceneH $^{+}$ & $\begin{array}{l}\text { Ground state } \\
\text { eneray/C1 }\end{array}$ & $\begin{array}{c}\mathrm{S}_{1} \text { vertical } \\
\text { transition energy }\end{array}$ & $\begin{array}{c}\mathrm{S}_{2} \text { vertical } \\
\text { transition energy }\end{array}$ & $\begin{array}{l}\mathrm{S}_{1} \leftarrow \mathrm{S}_{0} \text { adiabatic } \\
\text { transition energy }\end{array}$ \\
\hline $\mathrm{C} 1$ & 0 & 2.14 & 2.26 & 1.93 \\
\hline
\end{tabular}

For naphthalene the ground state retains a planar Cs symmetry and the excited state presents a local minimum in planar Cs symmetry for the two isomers.

For anthracene and tetracene the ground and the excited state geometries deviate slightly from Cs symmetry, the carbon atom linked to 2 hydrogen atoms coming slightly out of the molecular plane by $10^{\circ}$.

For the most stable isomers, the calculations show that the changes in geometry between the ground and the first excited state are not very large and Franck Condon factors to the 0-0 transition can be expected to be reasonable: this corroborates the assignment of the first band observed in each spectrum to the 0-0 transition.

The values of the transition energies calculated and observed for the protonated molecules are displayed in figure 3 as a function of the number of aromatic rings and compared with the transition energies of the neutral linear $\mathrm{PAH}^{20-23}$. 


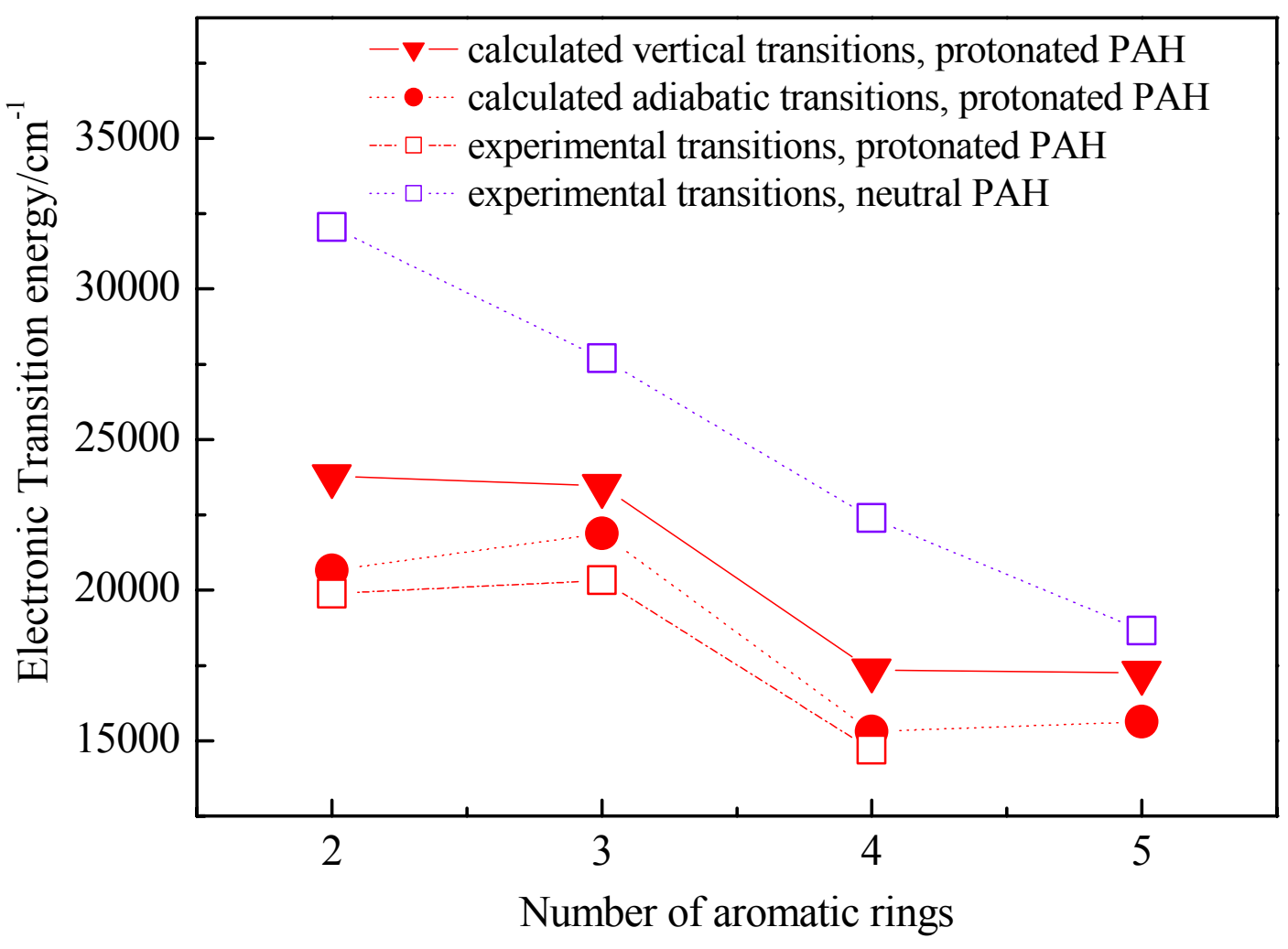

Figure 3. Variation of the first $S_{1} \leftarrow S_{0}$ electronic transition of linear PAHs with the number of aromatic rings. open blue squares:, experimental transitions of neutral $P A H s^{20-23}$; filled red triangles: calculated vertical transitions for protonated PAHs; filled red circles: calculated adiabatic transition for protonated PAHs; open red squares: experimental band origin of protonated PAHs.

\section{Discussion}

Comparison between experiment and calculated transitions.

As can be seen in table 1, the agreement between the calculated adiabatic transition energy for the most stable isomers and the experiment is quite good, while the vertical transition energies do not agree with the experimental observations. This shows that the excited state optimization is a necessary step to get reasonable agreement and that the RI-CC2 method gives reliable results at a reasonable computing cost for these systems. For the smaller systems (i.e naphthalene and anthracene), the agreement is even better when the variation of the ZPE between the ground and excited state is taken into account. This requires the calculation of the $S_{1}$ and $S_{0}$ vibrational frequencies and the $\delta Z P E$ values calculated are 0.12 $\mathrm{eV}$ for naphthalene and $0.18 \mathrm{eV}$ for anthracene. 
It should be noticed that the very good agreement between the observed spectrum and the calculated adiabatic transition energy is only obtained for the most stable isomer. In anthracene and tetracene the electronic transitions of the other isomers are shifted by more than $0.8 \mathrm{eV}$ and cannot account for the observed spectra, which indicates that electronic spectroscopy can be useful to differentiate ground state isomers.

In the case of naphthalene, the calculated adiabatic transition energies for the two isomers are close. It seems however that we see only one isomer, and since the $\mathrm{C} 1$ isomer is the most stable $\mathrm{e}^{1,5,24}$ and since the transition calculated with ZPE correction is very close to the experimental origin while the transition energy for the $\mathrm{C} 2$ isomer is lower, we assign the observed spectrum to the $\mathrm{C} 1$ isomer.

\section{Comparison between neutral and protonated molecules}

The electronic structure of the neutral and protonated molecules are quite similar having the same number of electron and both having a closed shell structure but their electronic spectra are quite different. For the neutral molecules, the electronic transition energies slowly decrease as the size of the molecules increases (figure 3). In contrast for the protonated ions, the transition energy does not show a monotonic behaviour. The anthracene transition is blue shifted as compared to the naphthalene transition and the tetracene transition is red shifted. The reason for this behaviour can be understood on the basis of ab-initio calculations. 

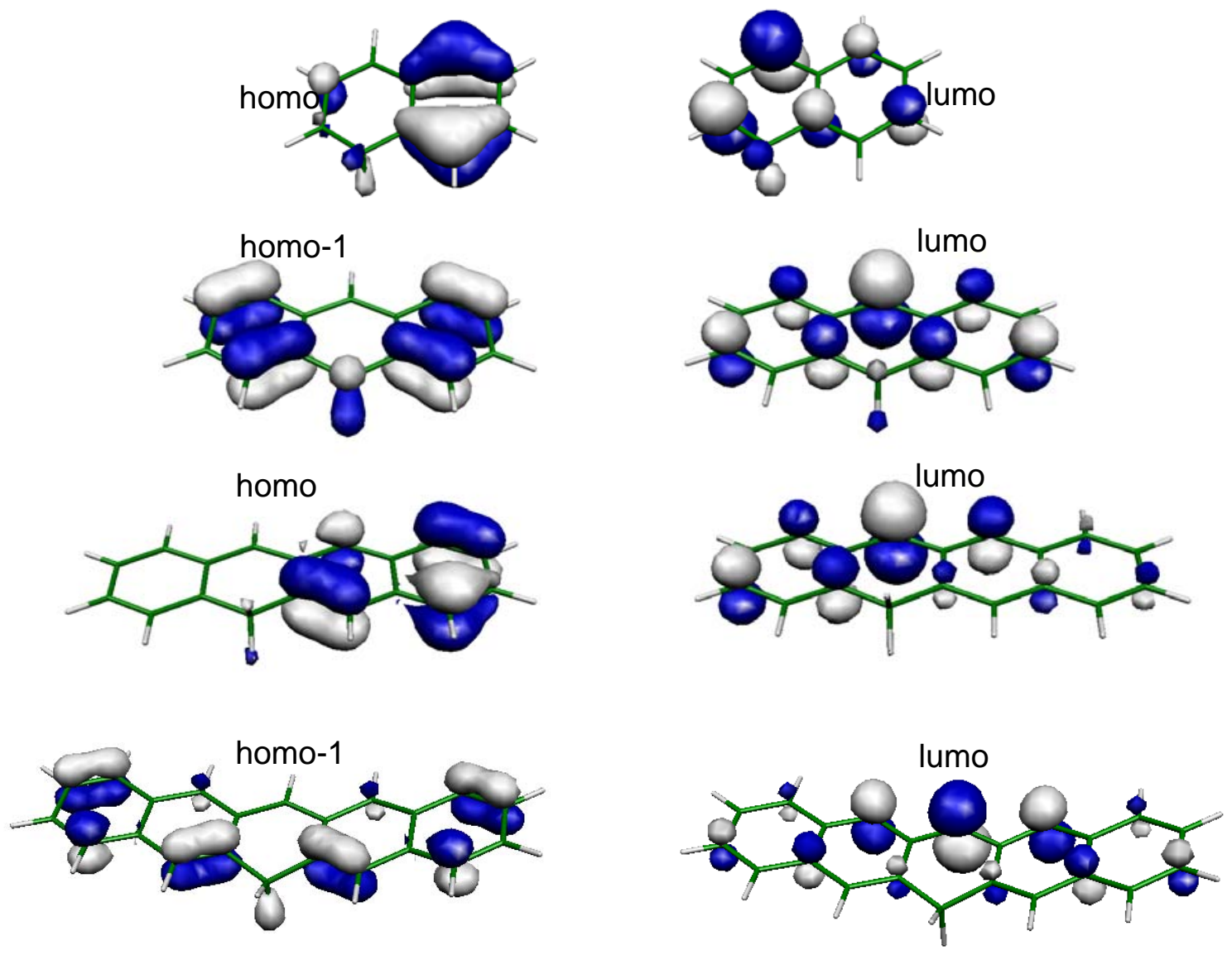

Figure 4: orbitals involved in the excited state transitions in protonated linear PAH.

In anthracene and pentacene, the lowest excited state corresponds to a transition from the HOMO-1 orbital towards the LUMO and for naphthalene and tetracene the lowest excited state involves a HOMO-LUMO transition. Figure 4 shows that, for an even number of aromatic rings, the excitation corresponds to the transition of an electron from the non protonated part of the molecule to the protonated part. This charge transfer character is very similar to what has been observed for the benzene dimer, in which the lowest excited state corresponds to the excitation of the electron from the neutral benzene toward the protonated benzene part. The reason why the charge transfer state is very low in energy in the case of the protonated benzene dimer can be understood with simple arguments: in a crude approximation, the HOMO-LUMO gap is not very different in the protonated and the neutral molecule. However, the HOMO orbital of benzene $\mathrm{H}^{+}$is significantly lower in energy, because the positive charge increases the ionisation energy due to larger Coulomb attraction. Thus, in the protonated benzene dimer, the HOMO is localized on the neutral benzene part, whereas the LUMO is localized on the benzene $\mathrm{H}^{+}$moiety. Consequently, the HOMO-LUMO electronic transition corresponds to a charge transfer state, which is lower in energy than the 
transitions localized on each of the constituents. The charge-transfer state of protonated benzene dimer leads to an intense absorption observed in the visible region. ${ }^{12}$ The situation is analogous in protonated naphthalene: the two parts of the molecule are no longer equivalent and there is a charge transfer from the neutral aromatic ring to the protonated moiety, leading to a large red shift of the transition as compared to neutral naphthalene. ${ }^{16}$ The results obtained for protonated tetracene indicate that this charge transfer also occurs in larger systems.

For molecules with an odd number of aromatic rings, in the most stable structure the proton is located on the central ring, with a symmetry plane perpendicular to the molecular rings and including the $\mathrm{CH}_{2}$ group (Cs molecular symmetry). Under this symmetry there is no charge transfer state and thus the excitation is more similar to the $\pi \pi *$ excitation of the neutral molecules which is higher in energy. This has already been observed for protonated benzaldehyde and seems to be quite general.

\section{Linear $(P A H) H^{+}$and Diffuse Interstellar Bands.}

$(\mathrm{PAH}) \mathrm{H}^{+}$have been suggested to be potential candidates for DIBs carriers. ${ }^{1,25-27}$, if smaller $(\mathrm{PAH}) \mathrm{H}^{+}$molecules are usually considered to be less favourable candidates, following photochemical stability arguments, larger ones may have a chance to be stable. It seems that there is no correspondence between the reported DIBs ${ }^{28}$ and the bands observed in the present experiment. Thus protonated naphthalene, anthracene and tetracene are not among the most intense DIBs carriers. Obviously, optical spectra of larger $(\mathrm{PAH}) \mathrm{H}^{+}$ions are needed for testing the DIBs hypothesis and corresponding experiments are currently underway.

At last, the quality of comparison between calculations and experiment allows predicting that the pentacene transition should be higher in energy than that of tetracene. It has not yet been possible to confirm this prediction since it seems very difficult to cool down this molecule. May be in the future with a better experimental setup we will be able to test this prediction.

Another point that will be addressed in the future is the properties of non linear PAHs which might be carriers of the diffuse interstellar bands.

\section{Conclusions}

We present the first experimental observation of electronic spectra of protonated linear PAHs. In contrast to the neutral molecules, the first electronic transitions are all in the visible region. The transition energies do not decrease monotonically with the size of the molecule as 
for their neutral counterparts, which can be understood with the help of $a b$ initio calculations by the presence of low lying charge transfer states in the protonated PAHs with an even number of aromatic rings.

\section{Acknowledgments}

The authors thank Pr. O. Dopfer for helpful discussion. This work has been supported by the Université Paris-Sud 11, by the ANR research grant (NT05-1 44224), and the PROCOPE 17832NK program. I.A. thanks the Atomic Energy Commission of Syria for financial support.

Supplementary Information: experimental set-up and procedures 


\section{References}

1. Lorenz, U. J.; Solca, N.; Lemaire, J.; Maitre, P.; Dopfer, O., Infrared spectra of isolated protonated polycyclic aromatic hydrocarbons: Protonated naphthalene. Angew. Chem. Int. Ed. 2007, 46, 6714-6716.

2. Solcà, N.; Dopfer, O., Protonated Benzene: IR Spectrum and Structure of C6H7+. Angew. Chem. Int. Ed. 2002, 41, 3628-3631.

3. Knorke, H.; Langer, J.; Oomens, J.; Dopfer, O., Infrared Spectra of Isolated Protonated Polycyclic Aromatic Hydrocarbon Molecules. Astrophys J. 2009, 706, L66-L70.

4. Zhao, D.; Langer, J.; Oomens, J.; Dopfer, O., Infrared spectra of protonated polycyclic aromatic hydrocarbon molecules:Azulene. J. Chem. Phys. 2009, 131, 184307.

5. Ricks, A. M.; Douberly, G. E.; Duncan, M. A., The Infrared Spectrum of Protonted Naphthalene and its Relevance for the Unidentified Infrared Bands. Astrophysical Journal 2009 702, (1), 301-306.

6. Rode, M. F.; Sobolewski, A. L.; Dedonder-Lardeux, C.; Jouvet, C.; Dopfer, O., Computational Study on the Photophysics of Protonated Benzene J. Phys. Chem. A 2009, 113, 5865-5873

7. Alata, I.; Omidyan, R.; Dedonder-Lardeux, C.; Broquier, M.; Jouvet, C., Electronically excited states of protonated aromatic molecules: benzaldehyde. Phys. Chem. Chem. Phys. 2009, 11, (48), 11479-11486.

8. Kang, H.; Jouvet, C.; Dedonder-Lardeux, C.; Martrenchard, S.; Gregoire, G.; Desfrancois, C.; Schermann, J. P.; Barat, M.; Fayeton, J. A., Ultrafast deactivation mechanisms of protonated aromatic amino acids following UV excitation. Phys. Chem. Chem. Phys. 2005, 7, (2), 394-398.

9. Gregoire, G.; Jouvet, C.; Dedonder, C.; Sobolewski, A. L., On the role of dissociative pi sigma* states in the photochemistry of protonated tryptamine and tryptophan: An ab initio study. Chem. Phys. 2006, 324, (2-3), 398-404.

10. Boyarkin, O. V.; Mercier, S. R.; Kamariotis, A.; Rizzo, T. R., Electronic Spectroscopy of Cold, Protonated Tryptophan and Tyrosine. J. Am. Chem. Soc. 2006, 128, 2816-2817.

11. Rizzo, T. R.; Stearns, J. A.; Boyarkin, O. V., Spectroscopic studies of cold, gas-phase biomolecular ions. Int. Rev. Phys. Chem. 2009, 28, (3), 481-515.

12. Chakraborty, S.; Omidyan, R.; Alata, I.; Nielsen, I. B.; Dedonder, C.; Broquier, M.; Jouvet, C., Protonated Benzene Dimer: An Experimental and Ab Initio Study. J. Am. Chem. Soc. 2009, 131, 11091-11097

13. Freiser, B. S.; Beauchamp, J. L., Photochemistry of organic ions in the gas phase. Comparison of the gas phase photodissociation and solution absorption spectra of benzoyl cation, protonated benzene, and protonated mesitylene. J. Am. Chem. Soc. 1976, 98, 31363139.

14. Perun, S.; Sobolewski, A. L.; Domcke, W., Ab initio studies on the radiationless decay mechanisms of the lowest excited singlet states of 9H-adenine. J. Am. Chem. Soc. 2005127 , (17), 6257-6265

15. Perun, S.; Sobolewski, A. L.; Domcke, W., Role of electron-driven proton-transfer processes in the excited-state deactivation adenine-thymine base pair. J. Phys. Chem. A 2006, 110 (29), 9031-9038

16. Alata, I.; Omidyan, R.; Broquier, M.; Dedonder, C.; Dopfer, O.; Jouvet, C., Effect of Protonation on the Electronic Structure of PAH Molecules. Phys. Chem. Chem. Phys. 2010, submitted. 
17. Ebata, T., Study on the Structure and Vibrational Dynamics of Functional Molecules and Molecular Clusters by Double Resonance Vibrational Spectroscopy. Bull. Chem. Soc. Jpn. 2009, 82, (2), 127-151.

18. Lucas, B.; Barat, B.; Fayeton, J. A.; Jouvet, C.; Çarçabal, P.; Grégoire, G., Statistical versus non statistical photofragmentation protonated GWG tri-peptide induced by UV excitation. Chem. Phys. 2008, 347, (1-3), 324-330.

19. NIST, In webbook of chemistry, http://webbook.nist.gov/chemistry/.

20. Cockett, M. C. R.; Ozeki, H.; Okuyama, K.; Kimura, K., Vibronic Coupling in the Ground Cationic State of Naphthalene - a Laser Threshold Photoelectron [Zero KineticEnergy (Zeke)-Photoelectron] Spectroscopic Study. J. Chem. Phys. 1993, 98, (10), 7763-7772. 21. Hoheisel, G.; Heinecke, E.; Hese, A., Optical-optical double resonance spectroscopy on pentacene molecules in a supersonic jet. Chem. Phys. Lett. 2003, 373, (3-4), 416-421.

22. Lambert, W. R.; Felker, P. M.; Syage, J. A.; Zewail, A. H., Jet Spectroscopy of Anthracene and Deuterated Anthracenes. J. Chem. Phys. 1984, 81, (5), 2195-2208.

23. Vanherpen, W. M.; Meerts, W. L.; Dymanus, A., Rotationally Resolved Laser Spectroscopy of Tetracene and Its Vanderwaals Complexes with Inert-Gas Atoms. J. Chem. Phys. 1987, 87, (1), 182-190.

24. Sebree, J. A.; Kislov, V. V.; Mebel, A. M.; Zwier, T. S., Spectroscopic and Thermochemical Consequences of Site-Specific H-Atom Addition to Naphthalene. J. Phys. Chem. A 2010, 114, 6255-6262.

25. Snow, T.; Page, L. V.; Keheyan, Y.; Bierbaum, V. M., The interstellar chemistry of PAH cations. Nature 1998, 391, 259-260.

26. Pathak, A.; Sarre, P. J., Protonated PAHs as carriers of diffuse interstellar bands. Mon. Not. R. Astron. Soc.:letters 2008, 391, L10-L14.

27. Hammonds, M.; Pathak, A.; Sarre, P. J., TD-DFT calculations of electronic spectra of hydrogenated protonated polycyclic aromatic hydrocarbon (PAH) molecules: implications for the origin of the diffuse interstellar bands? Phys. Chem. Chem. Phys. 2009, 11, 4458-4464.

28. Jenniskens, P.; Desert, F. X., A survey of diffuse interstellar bands (3800-8680ANGSTROM). Astron. Astrophys. Suppl. Ser. 1994, 106, 39-78. 
TOC

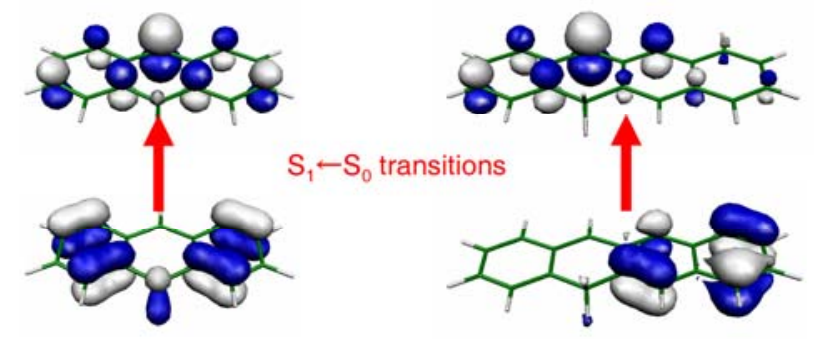

